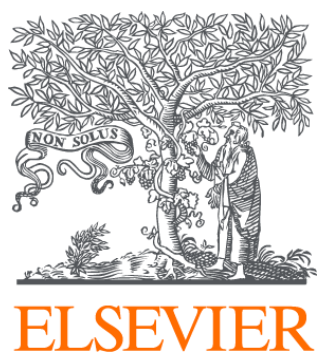

Since January 2020 Elsevier has created a COVID-19 resource centre with free information in English and Mandarin on the novel coronavirus COVID-

19. The COVID-19 resource centre is hosted on Elsevier Connect, the company's public news and information website.

Elsevier hereby grants permission to make all its COVID-19-related research that is available on the COVID-19 resource centre - including this research content - immediately available in PubMed Central and other publicly funded repositories, such as the WHO COVID database with rights for unrestricted research re-use and analyses in any form or by any means with acknowledgement of the original source. These permissions are granted for free by Elsevier for as long as the COVID-19 resource centre remains active. 
Original article

\title{
Examination of the relationship between emergency department presentations and population mortality: a multicenter analysis of emergency department presentations during the COVID-19 pandemic
}

\author{
Monica Solbiati ${ }^{\mathrm{a}, \mathrm{b}}$, Lorenzo Russo ${ }^{\mathrm{a}}$, Anna Beverina ${ }^{\mathrm{b}}$, Paolo Groff ${ }^{\mathrm{c}}$, Andrea Strada ${ }^{\mathrm{d}}$, \\ Ludovico Furlan $^{\mathrm{a}}$, Nicola Montano ${ }^{\mathrm{a}, \mathrm{b}}$, Giorgio Costantino ${ }^{\mathrm{a}, \mathrm{b}, *}$, Giovanni Casazza ${ }^{\mathrm{b}}$ \\ a Fondazione IRCCS Ca' Granda Ospedale Maggiore Policlinico, Milan, Italy \\ ${ }^{\mathrm{b}}$ Università degli Studi di Milano, Milan, Italy \\ ${ }^{\mathrm{c}}$ Pronto Soccorso e Osservazione Breve, Azienda Ospedaliera di Perugia, Perugia, Italy \\ ${ }^{\mathrm{d}}$ Medicina d'Emergenza Urgenza, Azienda Ospedaliero Universitaria S. Anna, Ferrara, Italy
}

\section{A R T I C L E I N F O}

\section{Keywords:}

Covid 19

Emergency department

Appropriateness

Hospitalization

Mortality

\begin{abstract}
A B S T R A C T
Background: : In the spring of 2020, Italy experienced a significant reduction in the number of emergency department (ED) presentations during the first wave of the COVID-19 pandemic. If ED access has an impact on patients' prognosis, such a reduction in ED presentations would be expected to correlate with a parallel increase in the mortality rate of the corresponding population. The aim of the present study was to evaluate the impact of reduced ED presentations on the all-cause mortality of the general population.

Methods: Absolute and relative variation in ED accesses from March 1 to April 30 of both 2019 and 2020 in three hub hospitals in areas with different COVID-19 prevalence and age-standardized mortality data from January 1 to June 30 in 2019 and 2020 of the same areas were evaluated.

Results: : During March and April 2020, ED consults were decreased of approximately 50\% in all three hospitals, as compared with the same months in 2019. There was a marked increase in cumulative mortality in Milan (high SARS-CoV2 infection spread zone) compared with the same period in 2019. In the other two municipalities (Ferrara and Perugia), which had intermediate and low levels of infection spread, the mortality in 2020 was not substantially changed from that of 2019.

Conclusions: : Taking into account the increase in mortality due to SARS-CoV-2, reductions in ED access did not seem to affect death rates. If this finding will be confirmed, ED organization and access would need to be reconsidered.
\end{abstract}

\section{Introduction}

The first deaths outside of China due to COVID-19 (coronavirus disease 2019), caused by the SARS-CoV-2 (severe acute respiratory syndrome coronavirus 2), occurred in February 2020 [1]. Subsequently, there was widespread rapid transmission of the virus internationally, with a particularly challenging surge of COVID-19 cases and deaths in Italy [2-5], leading the World Health Organization to recognize the outbreaks officially as a pandemic in early March [6].

Although the North-West of Italy was one of the most impacted regions in the spring of 2020, almost all Italian national territory hospitals have experienced a significant reduction in the number of emergency department (ED) presentations [7-9], even in areas of low SARS-CoV-2 prevalence. This situation provides an exceptional opportunity to study the role of the ED in current healthcare systems [10]. Such a reduction in ED presentations would be expected to correlate with a parallel increase in the mortality rate of the corresponding population. If these expected results are not found, the appropriateness of ED presentations should be questioned. The present study aimed to evaluate the impact of reduced ED presentations on the all-cause mortality of the general population in Italy during the first wave of the COVID-19 pandemic.

\footnotetext{
* Corresponding author at: IRCCS, Fondazione Ca' Granda, Medicina Interna, via Francesco Sforza 34, Milano, Italy.

E-mail address: giorgio.costantino@unimi.it (G. Costantino).
} 


\section{Methods}

\subsection{Study design}

A multicentric observational study was conducted. We collected data on ED presentations from three centers located in three regions within Italy with different levels of SARS-CoV-2 infection spread. For each area, we compared mortality data in the first semester of 2019 and 2020.

\subsection{Population and study center selection}

We classified each Italian province according to the severity of SARSCoV-2 infection spread, based on COVID-19 mortality data provided by the Italian National Institute of Statistics (ISTAT) and the National Institute of Health (Supplement), which provide daily mortality data for the 21 Italian regions to the public [11]. Based on tertiles of COVID-19 age-standardized mortality (first tertile 9.5/100,000; second tertile 46.8/100,000), we classified the provinces into three categories: green (low mortality), yellow (intermediate mortality), and red (high mortality) .

We selected three Italian provinces (one from each area): Milan (red), Ferrara (yellow), and Perugia (green). We then selected one hospital center for each province: Ospedale Maggiore Policlinico of Milan, Azienda Ospedaliero-Universitaria di Ferrara (Arcispedale Sant'Anna), and Azienda Ospedaliera di Perugia (Ospedale Santa Maria della Misericordia), respectively. These hospitals were chosen because they are considered to be primary hubs in their respective provinces. This characteristic is especially valid for the Perugia and Ferrara hospitals, whereas Policlinico of Milan is part of a wide integrated hospital network. We also considered convenience criteria (i.e., a prior professional relationship with the aforementioned hospitals), which simplified the data collection process.

The total number of hospital beds available, number of ED yearaccesses, and rate of hospital admissions for each center are as follows:

1 Ospedale Maggiore Policlinico (Milan, 1390000 inhabitants): 900 beds; 109,000 ED consults, and 36,000 inpatient admissions in 2019.

2 Arcispedale Sant'Anna (Ferrara, 132000 inhabitants): 710 beds; 90,000 ED consults, and 27,000 inpatient admissions in 2019.

3 Ospedale Santa Maria della Misericordia (Perugia, 164000 inhabitants): 759 beds; 60,000 ED consults, and 35,000 inpatient admissions in 2019.

\subsection{Data extraction}

\subsubsection{ED data}

We analyzed all patients that were evaluated in one of the three included EDs from March 1 to April 30 of both 2019 and 2020. Data on the number of ED presentations, triage priority codes given, and number of inpatient admissions from the ED were extracted from electronic charts and tabulated in an Excel database. Pediatric, gynecological, and fast track (dermatology, otolaryngology, and ophthalmology) accesses were excluded from the analysis.

\subsubsection{Mortality}

All-cause mortality data for each included municipality (Milan, Ferrara, and Perugia) were obtained from the ISTAT database. Because a reduction in ED access may affect mortality over a medium-length period, we analyzed mortality data from January 1 to June 30 in 2019 and 2020 .

\subsubsection{Statistical analysis}

ED data are reported as counts and percentages. We calculated the absolute and relative variation in ED access and hospitalization from 2019 to 2020 for the three included hospitals. For mortality data analyses, we retrieved the daily number of all-cause deaths and calculated the cumulative absolute mortality from January 1 to June 30 for years of 2019 and 2020. Mortality data are reported in cumulative frequency diagrams. Chi-square test was used to assess if variation over time of low-priority codes (white and green) was different from variation of high-priority codes (yellow and red). P values lower than 0.05 , two sided, were considered statistically significant.

Analyses were performed using the SAS (release 9.4) statistical software.

\section{Results}

During March and April 2020, ED consults were decreased in all three analyzed EDs compared with the same months in 2019. The decrease was homogeneous across the three hospitals, with an overall reduction of approximately 50\%. Quantities of ED visits at these hospitals during 2019 and 2020 are reported in Table 1 according to triage code. A reduction in access was observed for all triage codes and was higher for low-priority codes. This reduction in ED presentations occurred before March 8 (the start of the lockdown in Italy) (eTable 1). When comparing reductions in low- versus high-priority codes, a statistical significant difference was observed ( $\mathrm{p}<0.001$, for each hospital), showing a higher reduction in low-priority codes in 2020 as compared to 2019.

As shown in Table 2, the total number of ED presentations was lower in 2020 than in 2019, although the rate of hospitalization (upon ED presentations) was higher in 2020. This increase was more prominent in areas with intermediate (Ferrara) and high (Milan) levels of SARS-CoV-2 infection transmission.

Cumulative mortalities (January-June 2019 and 2020) in the metropolitan regions of Milan, Ferrara, and Perugia are shown in Fig. 1. Note that, there was a marked increase in mortality in Milan (red area, high SARS-CoV2 infection spread zone). In particular, for April 30, 2020 (when daily mortality rates returned to values similar to those of 2019), we observed a $46 \%$ increase in mortality (cumulative deaths: 6,563) compared with the same period in 2019 (cumulative deaths: 4,506). In the other two municipalities (Ferrara and Perugia), which had intermediate and low levels of infection spread, the mortality in 2020 was not substantially changed from that of 2019.

Table 1

Numbers of ED consults by triage priority color code from March 1 to April 30 in 2019 and 2020.

\begin{tabular}{lllll}
\hline Center & Code & $\begin{array}{l}\text { ED consults in } \\
2019, \mathrm{~N}(\%)\end{array}$ & $\begin{array}{l}\text { ED consults in } \\
2020, \mathrm{~N}(\%)\end{array}$ & $\begin{array}{l}\text { Relative } \\
\text { variation 2020 vs. } \\
2019\end{array}$ \\
\hline \multirow{2}{*}{ Milan } & Red & $437(5.4 \%)$ & $270(7.9 \%)$ & $-38 \%$ \\
& Yellow & $1596(19.7 \%)$ & $870(25.4 \%)$ & $-45 \%$ \\
& Green & $5740(70.8 \%)$ & $2221(64.9 \%)$ & $-61 \%$ \\
& White & $340(4.2 \%)$ & $59(1.7 \%)$ & $-83 \%$ \\
& Total & $8113(100.0 \%)$ & $3420(100.0 \%)$ & $-58 \%$ \\
Ferrara & & & & \\
& Red & $499(4.5 \%)$ & $297(5.5 \%)$ & $-40 \%$ \\
& Yellow & $3915(35.4 \%)$ & $2374(44.1 \%)$ & $-39 \%$ \\
& Green & $6260(56.7 \%)$ & $2536(47.1 \%)$ & $-59 \%$ \\
& White & $370(3.4 \%)$ & $176(3.3 \%)$ & $-52 \%$ \\
& Total & 11044 & $5383(100.0 \%)$ & $-51 \%$ \\
& & $(100.0 \%)$ & & \\
Perugia & & & $-36 \%$ \\
& Red & $259(2.6 \%)$ & $166(4.0 \%)$ & $-44 \%$ \\
& Yellow & $1798(18.3 \%)$ & $1003(24.2 \%)$ & $-60 \%$ \\
& Green & $6944(70.7 \%)$ & $2766(66.8 \%)$ & $-75 \%$ \\
& White & $823(8.4 \%)$ & $206(5.0 \%)$ & $-58 \%$ \\
& Total & $9824(100.0 \%)$ & $4141(100.0 \%)$ & -50
\end{tabular}

${ }^{a}$ Relative variation in the absolute number of ED consults, calculated as (2020 value - 2019 value) / (2019 value), inclusive of the months of March and April. 
Table 2

Hospitalization rate for ED accesses from March 1 to April 30 in 2019 and 2020.

\begin{tabular}{|c|c|c|c|c|c|c|}
\hline \multirow[t]{2}{*}{ Center } & \multicolumn{2}{|l|}{2019} & \multicolumn{2}{|l|}{2020} & \multicolumn{2}{|c|}{ Relative change 2020 vs. 2019} \\
\hline & ED consults & Hospitalizations N (\%) & ED consults & Hospitalizations N (\%) & ED consults & Hospitalizations \\
\hline Milan & 8113 & $1198(14.8 \%)$ & 3420 & $1043(30.5 \%)$ & $-58 \%$ & $106.5 \%$ \\
\hline Ferrara & 11044 & $2177(19.7 \%)$ & 5383 & $1860(34.6 \%)$ & $-51 \%$ & $75.3 \%$ \\
\hline Perugia & 9824 & $2259(23.0 \%)$ & 4141 & $1396(33.7 \%)$ & $-58 \%$ & $46.6 \%$ \\
\hline
\end{tabular}

a Calculated as (2020 value - 2019 value) / (2019 value), inclusive of the months of March and April.

\section{Discussion}

The present study showed that the total number of ED presentations decreased during the COVID-19 pandemic irrespective of local SARSCoV-2 transmission rates. This situation provided an exceptional opportunity to conduct a study to assess the effect of a dramatic reduction in ED presentations on short term mortality. This allowed to study the role of the ED in current healthcare systems. In the two regions without high transmission, we did not observe corresponding increase in overall mortality in the examined municipalities. Mortality increased only in the region with high SARS-CoV-2 infection spread (Milan). These data suggest that the reduction in ED presentations does not seem to affect mortality in the general population.

The presently documented reductions in ED presentations could have several causes. First, on March 8, the Italian government declared a national lockdown to mitigate local outbreaks. In addition, institutions asked the citizens repeatedly to avoid unnecessary ED access, both to prevent the risk of infection spread in crowded environments and to facilitate prompt care for those with true emergency needs. However, our data show that ED presentations began to decrease during the first days of March, before the lockdown. This finding suggests that one of the primary causes for the reduced ED presentations may have been people's fear of virus exposure in the ED. Notably, we observed reduced access for all triage codes, indicating that even people at potentially high medical risk were less likely to seek medical attention in the ED. Several recent reports have shown significant reductions in ED visits for major complaints, such as myocardial infarction, during the COVID-19 pandemic [12]; and such reductions have also been related to an increased mortality rate [13].

It is reasonable to expect that a reduction in ED access would be followed by an increase in mortality. Surprisingly, the mortality rates in the observed areas were similar to those of the previous year, except for Milan, where the high SARS-CoV2 infection spread may have contributed to an increased mortality. Hence, dramatic reductions in ED presentations $(>50 \%)$ in Italy were not followed by an increase in shortterm mortality. Such an unexpected finding has already been observed regarding the utility of hospitalization in older adults and it is an opportunity to critically rethink our health care system organization [14].

We observed an increase in the hospitalization rate of ED patients from 2019 to 2020 . This result suggests that regardless of triage code, the severity of ED cases was generally higher in 2020 than in 2019. Because the total number of hospital admissions was lower in 2020 than in 2019 , this observation is probably due to a greater reduction in lowseverity code patients who did not require hospitalization being evaluated in the ED.

All of these observations raise questions about the appropriateness of ED consults. An ED should aim to provide care primarily to patients affected by an injury or disease at risk of rapid deterioration of clinical status. If this aim is not being met by an emergency service, then the mortality in the area served by that service should increase rapidly. Our data showed that excluding the effects of COVID-19 (i.e., increased mortality in areas with high SARS-CoV-2 transmission), a reduction in ED presentations and in the absolute number of hospital admissions from the ED was not followed by a generalized increase in short-term mortality. Many reports have suggested that there may be substantial amounts of unnecessary ED visits [15]. However, in our study, we observed a reduction in all triage codes, indicating that fewer patients with more severe conditions presented to the ED [16-19].

Notably, the healthcare system in Italy is universal and, in most cases, free of charge. Thus, the absence of the expected mortality increase may be primarily due to a reduction in patients accessing the ED for a non-urgent problem. Indeed, it is known that ED crowding is associated with increased in-hospital mortality [20]. Therefore, the reduction in low-priority codes might have been related with a better care of patients requiring urgent care [21]. Other factors could be a reduction in adverse events caused by elective admission or a reduction in ED boarding, which could have led to better quality of care for patients affected by time-dependent diseases [10].

We cannot exclude the possibility that the lack of an increased mortality in areas with lower SARS-CoV-2 spread could be due, at least in part, to several interacting factors with diverse effects on the mortality of the general population. For example, the restrictive measures of the national lockdown may have reduced mortality indirectly by reducing traumatic injuries (e.g., workplace and transportation accidents) and pollution; such reductions may have offset increases in mortality due to reduced numbers of ED visits. However, the overall contribution of these causes to total acute mortality is low [22-24]. In any case, our results suggest that the introduction of a barrier to ED access had no effect on short-term mortality. Based on these results, stakeholders should reconsider how emergency services in Italy are currently being used. It is possible that the introduction of some form of barrier to ED access could improve ED efficiency without leading to substantial adverse events or increased mortality.

Our study has several limitations, the most important of which is the observational nature of the study. To limit confounding factors that we cannot control, a randomized controlled trial would be the most appropriate study design. However, for ethical and practical reasons, this type of study is not possible. For practical reasons, we analyzed data from only one hospital per area. We assumed that the selected hospitals would be representative of all hospitals within the same area and thus that the observed results would be generalizable. We acknowledge that this selection procedure may have introduced a bias. Future studies in which more hospitals are enrolled could help to strengthen the results. Moreover, it would have been interesting to analyze the specific causes of death to exclude other possible causes for increased mortality beyond SARS-CoV2 infection. However, reliable data on the cause of death was not available for 2020. Nevertheless, as we observed an increase in mortality only in high SARS-CoV2 prevalence area, we believe that such an increase is mainly due to the infection.

Finally, the true SARS-CoV2 infection prevalence is difficult to estimate because prevalence data are strongly influenced by the level of SARS-CoV-2 testing performed in the population. In addition, we classified geographical areas as high/medium/low infection spread according to official COVID-19 mortality statistics. This approach may have led to a misclassification of the areas included in the study. Nevertheless, it is reasonable to believe that mortality is not highly dependent on the ability to identify suspected cases (i.e., the number of SARS-CoV-2 tests performed).

\section{Conclusions}

ED visits were found to be markedly decreased during the COVID-19 


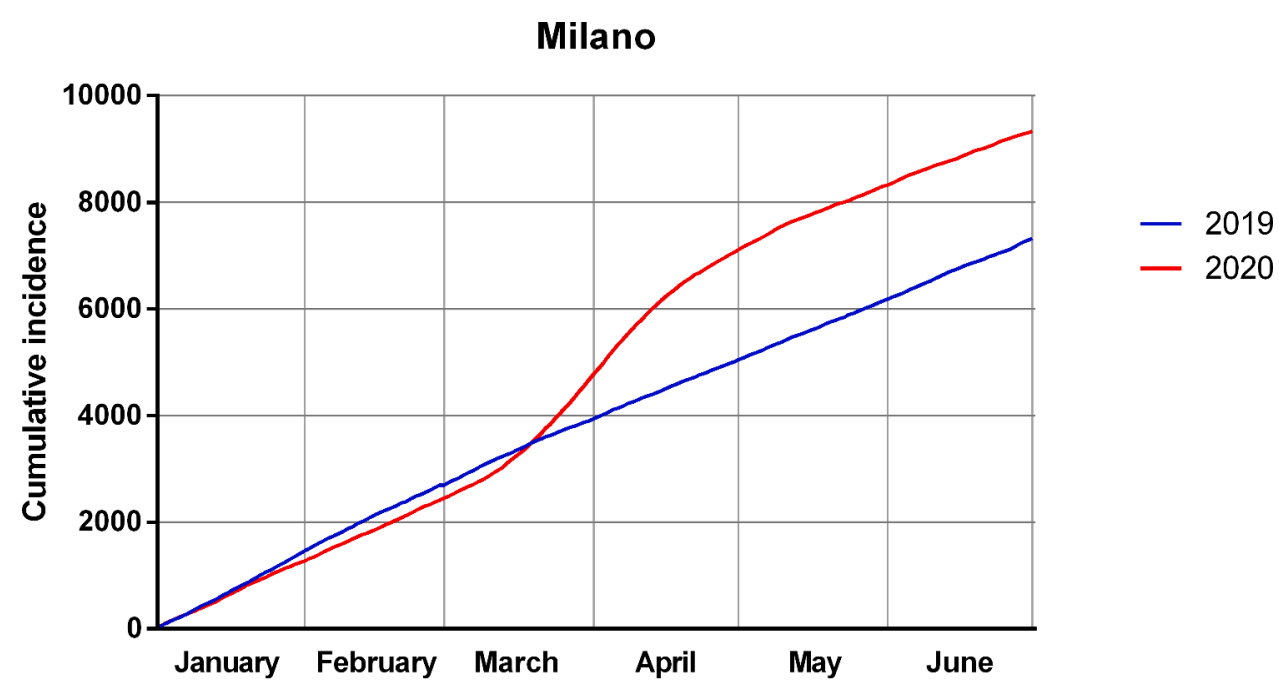

Ferrara

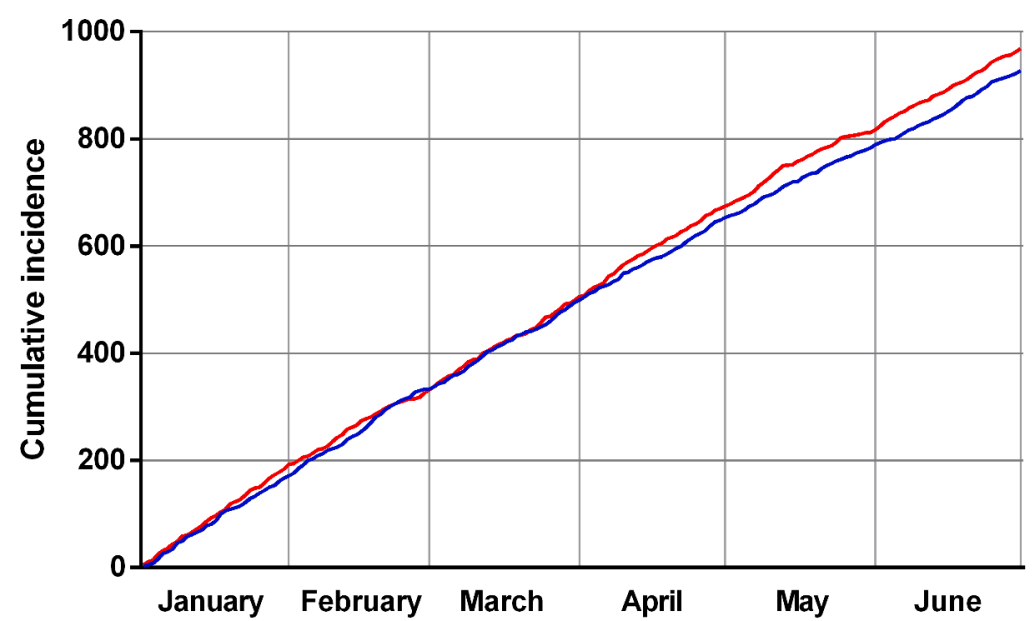

- 2019

- 2020

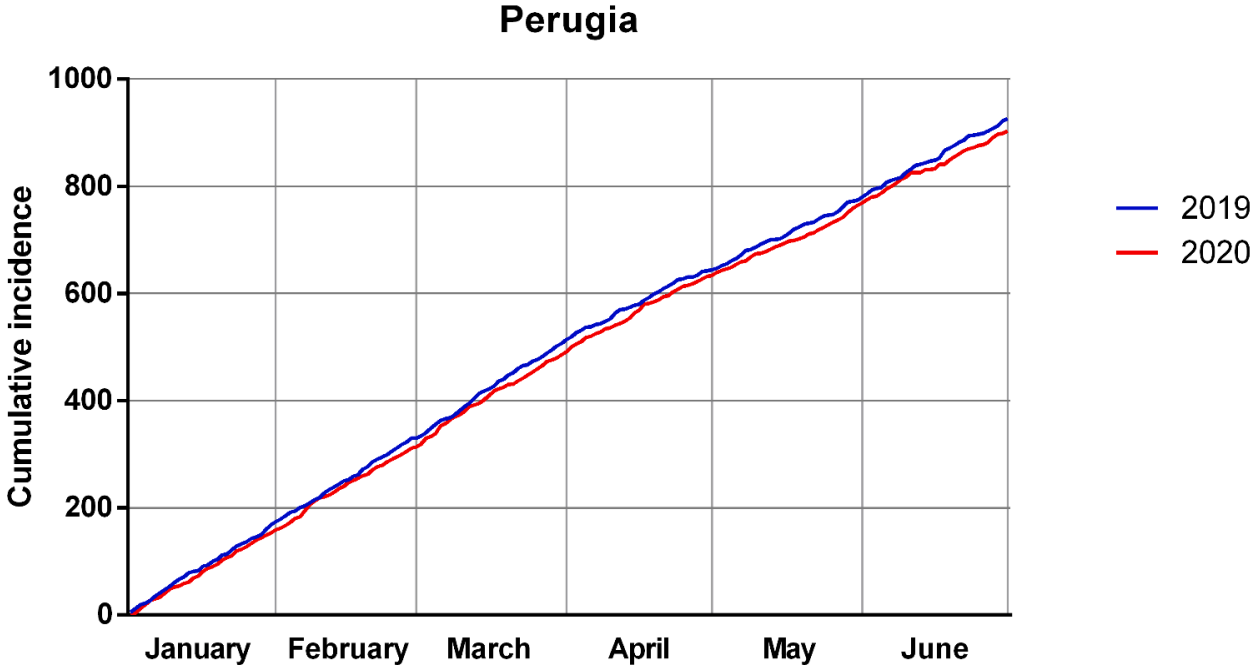

Fig. 1. Cumulative mortality from January 1 to June 30 in 2019 and 2020. 
epidemic across three regions of Italy with different levels of impact from the COVID-19 pandemic. The reduction was more pronounced for low-priority code cases, but a reduction was also evident for highpriority code cases. Taking into account the increase in mortality due to SARS-CoV-2, reductions in ED access did not seem to affect death rates. If this finding will be confirmed, ED organization and access would need to be reconsidered.

\section{Supplementary materials}

Supplementary material associated with this article can be found, in the online version, at doi:10.1016/j.ejim.2021.08.004.

\section{Bibliography}

1] Spiteri G, Fielding J, Diercke M, Campese C, Enouf V, Gaymard A, et al. First case of coronavirus disease 2019 (COVID-19) in the WHO European Region, 24 January to 21 February 2020. Eurosurveillance 2020;25. https://doi.org/10.2807/1560 7917.ES.2020.25.9.2000178.

[2] Perlini S, Canevari F, Cortesi S, Sgromo V, Brancaglione A, Contri E, et al. Emergency department and out-of-hospital emergency system (112-AREU 118) integrated response to Coronavirus Disease 2019 in a Northern Italy centre. Intern Emerg Med 2020;15:825-33. https://doi.org/10.1007/s11739-020-02390-4.

[3] Grasselli G, Pesenti A, Cecconi M. Critical care utilization for the COVID-19 outbreak in Lombardy, Italy: early experience and forecast during an emergency response. JAMA - J Am Med Assoc 2020;323:1545-6. https://doi.org/10.1001/ jama.2020.4031.

[4] Ferrari R, Maggioni AP, Tavazzi L, Rapezzi C. The battle against COVID-19: Mortality in Italy. Eur Heart J 2020;41:2050-2. https://doi.org/10.1093/ eurheartj/ehaa326.

[5] Signorelli C, Scognamiglio T, Odone A. COVID-19 in Italy: Impact of containmen measures and prevalence estimates of infection in the general population. Acta Biomed 2020;91:175-9. https://doi.org/10.23750/abm.v91i3-S.9511.

[6] WHO Director-General's opening remarks at the media briefing on COVID-19 - 11 March 2020 n.d. https://www.who.int/director-general/speeches/detail/who-dire ctor-general-s-opening-remarks-at-the-media-briefing-on-covid- 19-11-march-2020 (accessed July 15, 2021)

[7] Garrafa E, Levaggi R, Miniaci R, Paolillo C. When fear backfires: emergency department accesses during the COVID-19 pandemic. Health Policy (New York) 2020;124:1333-9. https://doi.org/10.1016/j.healthpol.2020.10.006.

[8] Davico C, Marcotulli D, Lux C, Calderoni D, Terrinoni A, Di Santo F, et al. Where have the children with epilepsy gone? An observational study of seizure-related accesses to emergency department at the time of COVID-19. Seizure 2020;83: 38-40. https://doi.org/10.1016/j.seizure.2020.09.025.

[9] Castoldi L, Solbiati M, Costantino G, Casiraghi E. Variations in volume of emergency surgeries and emergency department access at a third level hospital in
Milan, Lombardy, during the COVID-19 outbreak. BMC Emerg Med 2021;21:59. https://doi.org/10.1186/s12873-021-00445-7.

[10] Kocher KE, Macy ML. Emergency department patients in the early months of the coronavirus disease 2019 (COVID-19) Pandemic - what have we learned? JAMA Heal Forum 2020;1:e200705. https://doi.org/10.1001/ jamahealthforum.2020.0705.

[11] ISTAT. Impatto Dell'epidemia COVID-19 Sulla Mortalità totale Della Popolazione Residente periodo Gennaio-Maggio 2020 n.d. https://www.istat.it/it/files/2020/ 07/Rapp Istat Iss 9luglio.pdf.

[12] Mafham MM, Spata E, Goldacre R, Gair D, Curnow P, Bray M, et al. COVID-19 pandemic and admission rates for and management of acute coronary syndromes in England. Lancet 2020;396:381-9. https://doi.org/10.1016/S0140-6736(20) 31356-8.

[13] De Rosa S, Spaccarotella C, Basso C, Calabrò MP, Curcio A, Filardi PP, et al. Reduction of hospitalizations for myocardial infarction in Italy in the COVID-19 era. Eur Heart J 2020;41:2083-8. https://doi.org/10.1093/eurheartj/ehaa409.

[14] Costantino G, Solbiati M, Elli S, Paganuzzi M, Massabò D, Montano N, et al. Utility of hospitalization for elderly individuals affected by COVID-19. PLoS One 2021;16: e0250730. https://doi.org/10.1371/journal.pone.0250730.

[15] Uscher-Pines L, Pines J, Kellermann A, Gillen E, Mehrotra A. Emergency department visits for nonurgent conditions: systematic literature review. Am J Manag Care 2013;19:47-59.

[16] Martin A, Martin C, Martin PB, Martin PA, Green G, Eldridge S. Inappropriate" attendance at an accident and emergency department by adults registered in local general practices: how is it related to their use of primary care? J Heal Serv Res Policy 2002;7:160-5. https://doi.org/10.1258/135581902760082463.

[17] Ismail SA, Gibbons DC, Gnani S. Reducing inappropriate accident and emergency department attendances: a systematic review of primary care service interventions. Br J Gen Pract 2013;63. https://doi.org/10.3399/bjgp13X675395.

[18] MacKichan F, Brangan E, Wye L, Checkland K, Lasserson D, Huntley A, et al. Why do patients seek primary medical care in emergency departments? An ethnographic exploration of access to general practice. BMJ Open 2017;7. https://doi.org/ 10.1136/bmjopen-2016-013816.

[19] Caporaletti P, Maragno M, Cosi M, Cutuli O, Ingrassia S, Cocorocchio A, et al. Overcrowding in Italian Emergency Department : the survey of " Settimana Nazionale del Pronto Soccorso 2017 " SIMEU. Ital J Emerg Med 2018:1-6.

[20] Sun BC, Hsia RY, Weiss RE, Zingmond D, Liang L-J, Han W, et al. Effect of emergency department crowding on outcomes of admitted patients. Ann Emerg Med 2013;61:605-11. https://doi.org/10.1016/j.annemergmed.2012.10.026. e6.

[21] Bernstein SL, Aronsky D, Duseja R, Epstein S, Handel D, Hwang U, et al. The effect of emergency department crowding on clinically oriented outcomes. Acad Emerg Med 2009;16:1-10. https://doi.org/10.1111/j.1553-2712.2008.00295.x.

[22] Center for Health Statistics N. National Hospital Ambulatory Medical Care Survey: 2017 Emergency Department Summary Tables 2017.

[23] DiMaggio CJ, Avraham JB, Lee DC, Frangos SG, Wall SP. The epidemiology of emergency department trauma discharges in the United States. Acad Emerg Med 2017;24:1244-56. https://doi.org/10.1111/acem.13223.

[24] Faust JS, Du C, Mayes KD, Li S-X, Lin Z, Barnett ML, et al. Mortality from drug overdoses, homicides, unintentional injuries, motor vehicle crashes, and suicides during the pandemic, March-August 2020. JAMA 2021. https://doi.org/10.1001/ jama.2021.8012. 